\title{
Elevation of Peripheral Blood CD34+ and Platelet Levels After Exercise With Cooling and Compression
}

\author{
Mark C. Callanan, M.D., Kevin D. Christensen, M.D., Hillary A. Plummer, Ph.D., A.T.C., \\ Johnny Torres, B.S., and Adam W. Anz, M.D.
}

\begin{abstract}
Purpose: To analyze the cellular response and chemokine profiles following exercise using cooling and blood flow restriction on the Vasper system. Methods: Healthy male patients between the ages of 20 and 39 years were recruited. Testing was performed on the Vasper system, a NuStep cross-trainer with concomitant 4-limb venous compression with proximal arm cuffs at $40 \mathrm{~mm} \mathrm{Hg}$ and proximal leg cuffs at $65 \mathrm{~mm} \mathrm{Hg}$. A cooling vest and cooling mat $\left(8.3^{\circ} \mathrm{C}\right)$ were used. A 7-minute warm-up followed by alternating 30- and 60-second sprints with 1.5 and 2 minutes of active recovery, respectively, between each sprint. Peripheral blood was drawn before exercise, immediately following exercise (T20), 10 minutes after the first post-exercise blood draw (T30), and then every 30 minutes (T60, T90, T120, T150, T180). A blood draw occurred at 24 hours' postexercise. Complete blood count, monoclonal flow cytometry for CD34+, and enzymelinked immunosorbent assay were used to analyze the samples. Results: Sixteen healthy male patients (29.5 \pm 4.5 years, $1.78 \pm 0.05 \mathrm{~m}, 83.7 \pm 11.4 \mathrm{~kg}$ ) were enrolled. There was an immediate, temporary increase in white blood cell counts, marked by an increase in lymphocyte differential $(38.3 \pm 6.5$ to $44.3 \pm 9.0 \%, P=.001)$, decrease in neutrophil differential $(47.8 \pm 6.6$ to $42.0 \pm 9.1 \%, P<.001)$, and platelets $\left(239.5 \pm 57.2\right.$ to $\left.268.6 \pm 86.3 \mathrm{~K} \cdot \mu \mathrm{L}^{-1}, P=.01\right)$. Monocytes significantly decreased from PRE to T90 $(9.8 \pm 1.1$ to $8.9 \pm 1.1 \mathrm{~K} / \mu \mathrm{L}, P<.001)$ and T120 $(8.9 \pm 1.1 \mathrm{~K} / \mu \mathrm{L}, P<$ $.0001)$. There was a significant increase in CD $34+$ cells $\left(3.9 \pm 2.0\right.$ to $5.3 \pm 2.8$ cells $\left.\bullet \mu \mathrm{L}^{-1}, P<.001\right)$. No detectable differences in measured cytokine levels of interleukin (IL)-10, IL-6, granulocyte-macrophage colony-stimulating factor , IL1ra, tumor necrosis factor- $\alpha$, or IL- 2 were observed. Conclusions: A significant elevation of peripheral blood CD $34+$ and platelet levels immediately following the exercise session was observed; however, there was no effect on peripheral circulation of IL-10, IL-6, IL-1ra, tumor necrosis factor- $\alpha$, or IL-2. Clinical Relevance: Exercise can be considered as a way to manipulate point-of-care blood products like platelet-rich plasma and may increase product yield.
\end{abstract}

I nterest in biologic treatments for orthopaedic conditions has grown significantly in recent years. There are a variety of biologic options available in current practice, with the 2 most popular being platelet-rich

From the Orthopedic Clinic, Shreveport, Louisiana (M.C.C.); Revere Health Orthopedics, Provo, Utah (K.D.C.); and Andrews Research $\theta$ Education Foundation (H.A.P.) and Andrews Institute for Orthopedics $\theta$ Sports Medicine (A.W.A.), Gulf Breeze, Florida (J.T.), U.S.A.

The authors report the following potential conflicts of interest or sources of funding: A.W.W. reports grants, personal fees, and other from Arthrex; grants from CGG Medical; and personal fees from Smith $\theta$ Nephew and Bioventus, during the conduct of the study. Full ICMJE author disclosure forms are available for this article online, as supplementary material.

Received June 15, 2020; accepted October 9, 2020.

Address correspondence to Hillary A. Plummer, Ph.D., A.T.C., Andrews Research $\theta$ Education Foundation, 1020 Gulf Breeze Parkway, Gulf Breeze, FL32561.E-mail:hplummer47@gmail.com

(C) 2020 THE AUTHORS. Published by Elsevier Inc. on behalf of the Arthroscopy Association of North America. This is an open access article under the CC BY-NC-ND license (http://creativecommons.org/licenses/by-nc-nd/4.0/). 2666-061X/201054

https://doi.org/10.1016/j.asmr.2020.10.003 plasma and bone marrow aspirate to augment surgical procedures and treat degenerative conditions such as osteoarthritis. ${ }^{1-3}$ Platelet-rich plasma and bone marrow aspirate are thought to participate in inflammation control, injury recovery, tissue healing, and ideally tissue regeneration. The term stem cell is commonly used, and the 4 cardinal properties of cells with stem potential are reproducibility, multipotentiality, mobility, and paracrine control. Cells with stem potential reside in multiple orthopaedic tissues and are mobilized in periods of injury. 4

Animal and human physiology studies have highlighted the potential for environmental mobilization of stem cells without the need for pharmaceuticals. ${ }^{6}$ There is evidence that heat, hypoxia, and cold can stimulate stem cells to mobilize to the peripheral circulation. ${ }^{7} \mathrm{An}$ increase in the peripheral mobilization of platelets as well as hematopoietic progenitor cells (HPCs) following vigorous exercise in humans also has been observed..$^{7-10}$ Previous literature also has demonstrated that exercise results in a rise in peripheral interleukin-1 
receptor antagonist (IL-1ra) levels, which are thought to potentiate the effects of mononuclear cells. ${ }^{11,12}$ These findings highlight a potential method for improving the collection of HPCs from the peripheral circulation with limited comorbidity through the use of vigorous exercise. $^{13}$

The Vasper system is a NuStep that uses a cooling vest to keep body temperature low and compression cuffs around the extremities to provide blood flow restriction. The addition of the cooling vest and compression cuffs may help to provide additional stress to the body, which may increase the mobilization of HPCs to the peripheral circulation. ${ }^{7}$ If increased mobilization of HPCs and platelets to the peripheral circulation occurs, it may drive clinical research to examine whether exercise improves the efficacy of point-of-care blood products and ultimately improves patient outcomes. The purpose of this study was to analyze the cellular response and chemokine profiles following exercise using cooling and blood flow restriction on the Vasper system. This could be a potentially less-invasive method to mobilize stem cells to optimize the physiology of recovering orthopaedic patients as well as to manipulate point-of-care blood and bone marrow products in orthopaedics. We hypothesized that an increase in CD34+ cells, platelets, white blood cells (WBCs), neutrophils, lymphocytes, interleukin (IL)-10, IL-6, IL-1 ra, tumor necrosis factor$\alpha$ (TNF- $\alpha$ ), and IL-2 would occur following exercise with the Vasper system.

\section{Methods}

\section{Participants}

Healthy male participants between the ages of 20 and 39 years were recruited. Participants were excluded if they were less than $37.5 \mathrm{~kg}$ or greater than $99 \mathrm{~kg}$. Participants also were excluded if they had a medical history of diabetes, autoimmune disorders, blood disorders, disorders requiring immunosuppression, cancer, an ongoing infectious disease, or significant cardiovascular, renal, hepatic, or pulmonary disease. Significant cardiovascular disease included aortic valve disease, a history of deep-vein thrombosis, venous insufficiency, or cold-induced syndromes such as Raynaud's or coldinduced urticaria. All participants were required to have no medical restrictions that prevented undergoing the 20-minute exercise sessions. All procedures were approved by the hospital's institutional review board (Baptist Hospital Pensacola Institutional Review Board; approval 872465). Before data collection, all testing procedures, risks, and benefits of the specific study were explained to each participant and written informed consent was obtained.

\section{Testing Sessions}

The participants arrived for testing between 6:00 and 10:00 AM in the morning after eating a breakfast of their own choice. Testing was performed on the Vasper NuStep T5XR recumbent cross-trainer (Vasper Systems, Mountain View, CA) (Fig 1). The participant wore a cooling vest that was set at a temperature of $8.3^{\circ} \mathrm{C}$ $\left(47^{\circ} \mathrm{F}\right)$. Blood flow restriction cuffs were applied bilaterally on the upper arm and they provided $40 \mathrm{~mm} \mathrm{Hg}$ of compression throughout exercise testing. Cuffs also were applied bilaterally on the upper legs and provided $65 \mathrm{~mm} \mathrm{Hg}$ of compression. The participants also sat on a cooling pad with a temperature of $8.3^{\circ} \mathrm{C}\left(47^{\circ} \mathrm{F}\right)$. Testing was performed with the participant barefoot because the foot panels of the machine also provided cooling.

The participants were placed on the machine for 20 minutes of exercising. The machine was set to the same protocol for all participants. Participants performed a 7minute warm-up on the Vasper machine at a selfselected pace. Once the warm-up was completed, the participants performed 6 sprint intervals, alternating 30 -second and 60-second sprints. Following the 30second sprints the participants had 1.5 minutes of active recovery before the 60 -second sprint. Two minutes of active recovery followed each 60 -second sprint (Fig 2). Participants were instructed to exert maximal effort during the sprint periods. If a participant reached self-described exertional fatigue and could not finish the entire exercise protocol, then blood was drawn at the time they could no longer continue.

\section{Blood Sampling}

On the day of testing, participants had a forearm intravenous catheter placed using standard sterile techniques by a physician or nurse. This catheter was used to obtain the initial and serial postexercise blood samples. After the initial blood draw, participants completed the exercise regimen. Blood samples were collected for a complete blood count (CBC), enzymelinked immunosorbent assay (ELISA), and flow cytometry. Sampling began immediately following exercise (T20), 10 minutes after the first postexercise blood draw (T30), and was repeated every 30 minutes (T60, T90, T120, T150, T180). The catheter was removed following the T180 blood draw. Participants returned 24-hour (T24 hours) after exercise for a final blood draw. Participants were instructed to avoid exercising during the 24-hour time period before the final blood draw.

A CBC with differential was performed for each blood sample. CBCs were performed on a Sysmex XN-1000 hematology analyzer (Lincolnshire, IL). ELISA was performed on all samples to detect cytokine levels, which are of interest to orthopaedic conditions, and included IL-10, IL-6, granulocyte-macrophage colonystimulating factor (GM-CSF), IL-1ra, TNF- $\alpha$, or IL-2. 
Fig 1. Vasper NuStep T5XR recumbent cross-trainer.

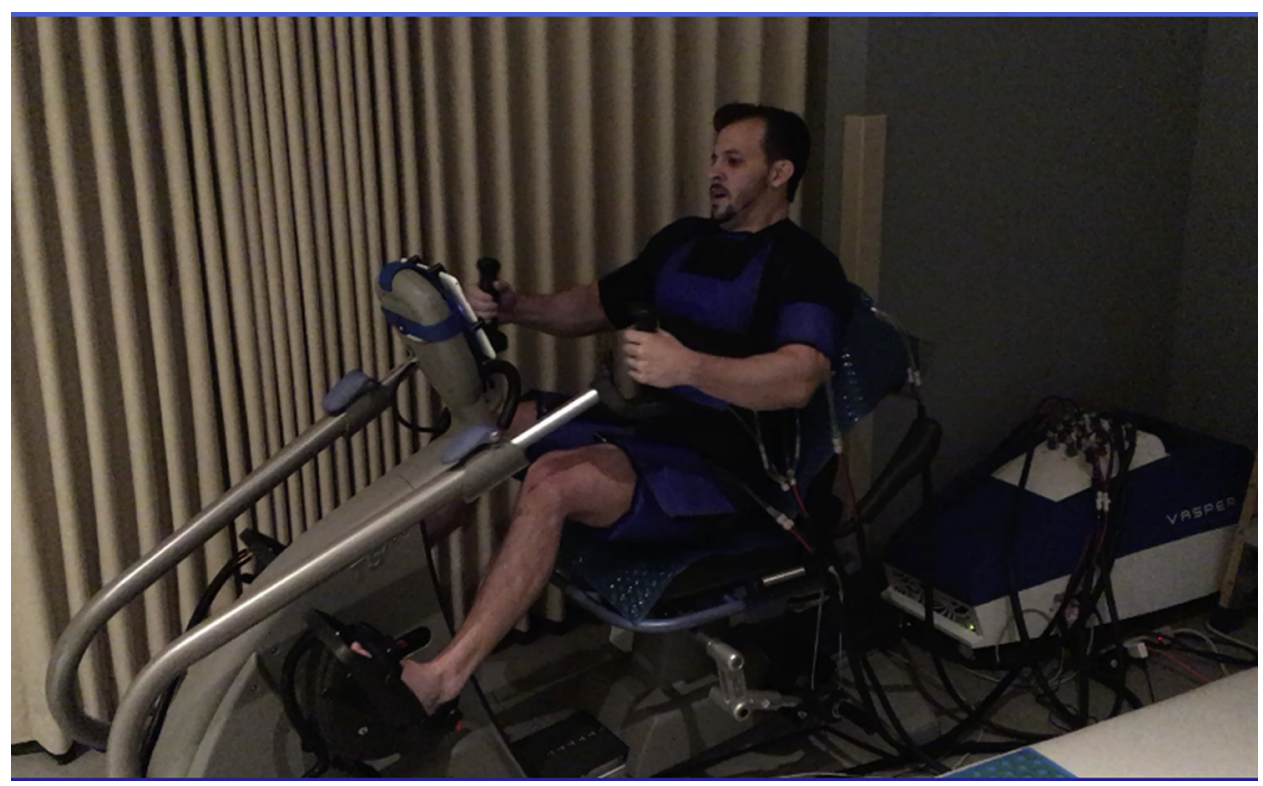

The MAGPIX (Luminex Corporation, Austin, TX) is a fluorescent-based instrument that uses charge-coupled device imaging technology. xMAP microspheres used for analysis are a family of fluorescently dyed carboxylated polystyrene microspheres that act as both the surface for the solution phase assay and the spectral identifier that the instrument detects and an detect multiple proteins in a single reaction.

Flow cytometry (Cytomics FC500 Flow Cytometer; Beckman Coulter Life Sciences, Indianapolis, IN) was used to quantify the amount of $\mathrm{CD} 34+$ peripheral blood cells. The samples were washed and diluted based on the sample concentration and then incubated for 30 minutes with a binding inhibitor. Following initial incubation, the samples were incubated for 30 minutes with antibodies for a CD34+ lineage panel. ${ }^{4}$ Standard isotype control samples were used. Each sample was washed, centrifuged, and then fixed with $10 \%$ formaldehyde before the flow cytometry analysis. The samples were only assessed at TPre and T20 due to the cost and the amount of time required to test each sample with flow cytometry.

\section{Statistical Analysis}

Analyses were performed on each variable with repeated-measures analysis of variance to test the hypothesis at a confidence level of $95 \%(P<.05)$. The results are presented as mean \pm standard deviation. Statistical significance was set a priori at $P<.05$. All analyses were conducted using IBM SPSS, version 25.0, software (IBM Corp. Armonk, NY). With an alpha level of $5 \%$ and a beta of $10 \%$ and an effect size of $200 \%$, a sample size of 10 was sufficient. This level of power has also been confirmed from previous mobilization studies. $^{4,8}$

\section{Results}

Sixteen healthy males $(29.5 \pm 4.5$ years, $1.78 \pm 0.05$ $\mathrm{m}, 83.7 \pm 11.4 \mathrm{~kg}$ ) were enrolled. One participant did not return for the 24-hour blood draw. Two
Fig 2. Exercise protocol that was used. S1-S6 indicate 30- and 60second sprint intervals.

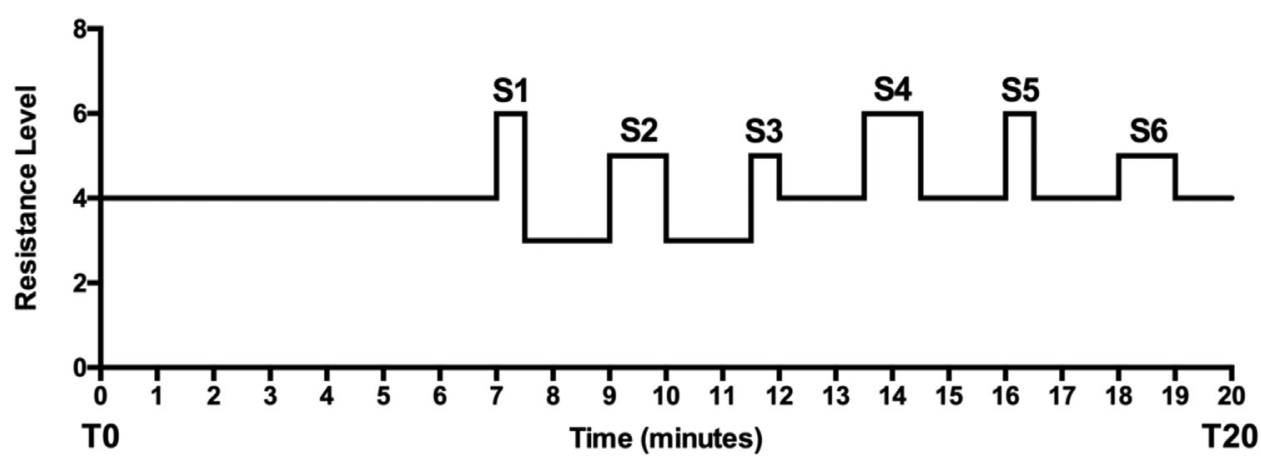


participants ELISA data were all outliers and were removed from the analysis. Data for platelets, WBCs, neutrophils, monocytes, lymphocytes, basophils, and eosinophils are presented in Table 1. ELISA data are presented in Table 2. CD34+ is presented in Figure 3. There was a significant increase in platelet counts from PRE to T20 $\left(239.5 \pm 57.2\right.$ to $268.6 \pm 86.3 \mathrm{~K} \cdot \mu \mathrm{L}^{-1}$, $\left.M_{\text {diff }}=29.1 \mathrm{~K} \cdot \mu \mathrm{L}^{-1}, P=.01\right)$. This was followed by a significant decrease in platelet levels from PRE noted at T60 $\left(239.5 \pm 57.2\right.$ to $219.3 \pm 64.9 \mathrm{~K} \cdot \mu \mathrm{L}^{-1}, \mathrm{M}_{\text {diff }}=$ $\left.-20.2 \mathrm{~K} \cdot \mu \mathrm{L}^{-1}, P=.007\right)$, T90 $\left(206.8 \pm 72.5 \mathrm{~K} \cdot \mu \mathrm{L}^{-1}\right.$, $\left.\mathrm{M}_{\text {diff }}=-32.7 \mathrm{~K} \cdot \mu \mathrm{L}^{-1}, P=.005\right), \mathrm{T} 120(225.6 \pm 53.7$ $\left.\mathrm{K} \cdot \mu \mathrm{L}^{-1}, \mathrm{M}_{\text {diff }}=-13.9 \mathrm{~K} \cdot \mu \mathrm{L}^{-1}, P=.02\right)$, and $\mathrm{T} 150$ $\left(213.9 \pm 71.6 \mathrm{~K} \cdot \mu \mathrm{L}^{-1}, \mathrm{M}_{\text {diff }}=-25.6 \mathrm{~K} \cdot \mu \mathrm{L}^{-1}, P=.01\right)$ and T180 $\left(204.0 \pm 62.9 \mathrm{~K} \cdot \mu \mathrm{L}^{-1}, \mathrm{M}_{\text {diff }}=-35.5 \mathrm{~K} \cdot \mu \mathrm{L}^{-1}\right.$, $P=.002)$. There was a significant increase in WBC counts from PRE to T20 $\left(6.1 \pm 1.1\right.$ to $8.8 \pm 2.7 \mathrm{~K} \cdot \mu \mathrm{L}^{-1}$, $\left.\mathrm{M}_{\text {diff }}=2.7 \mathrm{~K} \cdot \mu \mathrm{L}^{-1}, P<.001\right)$ and $\mathrm{T} 30(7.2 \pm 2.2$ $\left.\mathrm{K} \cdot \mu \mathrm{L}^{-1}, \mathrm{M}_{\text {diff }}=1.1 \mathrm{~K} \cdot \mu \mathrm{L}^{-1}, P=.02\right)$. A significant decrease was then observed in WBC counts noted at $\mathrm{T} 60\left(5.5 \pm 1.0 \mathrm{~K} \cdot \mu \mathrm{L}^{-1}, \mathrm{M}_{\text {diff }}=-0.6 \mathrm{~K} \cdot \mu \mathrm{L}^{-1}, P=.001\right)$ and T90 $\left(5.3 \pm 1.3 \mathrm{~K} \cdot \mu \mathrm{L}^{-1}, \mathrm{M}_{\text {diff }}=-0.8 \mathrm{~K} \cdot \mu \mathrm{L}^{-1}, P=\right.$ $.005)$. WBC counts plateaued until T180, where there was another significant increase from PRE levels $(7.1 \pm$ $\left.2.1 \mathrm{~K} \cdot \mu \mathrm{L}^{-1}, \mathrm{M}_{\text {diff }}=1.0 \mathrm{~K} \cdot \mu \mathrm{L}^{-1}, P=.04\right)$, followed by another significant decrease at T24 hours $(5.5 \pm 0.8$ $\left.\mathrm{K} \cdot \mu \mathrm{L}^{-1}, \mathrm{M}_{\text {diff }}=-0.6 \mathrm{~K} \cdot \mu \mathrm{L}^{-1}, P=.01\right)$.

There was a significant decrease in neutrophils (47.8 \pm 6.6 to $\left.42.0 \pm 9.1 \%, \mathrm{M}_{\text {diff }}=-5.8 \%, P=.001\right)$ and an increase in lymphocytes $(38.3 \pm 6.5$ to $44.3 \pm 9.0 \%$, $\mathrm{M}_{\text {diff }}=6.0 \%, P=.001$ ) initially from PRE out to T20. From T60 to T180, however, there was a significant increase in neutrophils T60 $\left(53.1 \pm 7.7 \%, \mathrm{M}_{\text {diff }}=5.3 \%, P\right.$ $<.001)$, T90 $\left(60.8 \pm 6.1 \%, \mathrm{M}_{\text {diff }}=13.0 \%, P<.001\right)$, $\mathrm{T} 120\left(66.6 \pm 7.5 \%, \mathrm{M}_{\text {diff }}=18.8 \%, P<.001\right), \mathrm{T} 150(67.8$ $\left.\pm 8.7 \%, \mathrm{M}_{\mathrm{diff}}=20.0 \%, P<.001\right), \mathrm{T} 180(68.0 \pm 9.3 \%$, $\left.\mathrm{M}_{\text {diff }}=20.2, P<.001\right)$. Conversely, there was an observed decrease in the peripheral lymphocyte levels during the same time period $\mathrm{T} 60\left(33.2 \pm 6.6 \%, \mathrm{M}_{\text {diff }}=\right.$ $-5.1 \%, P<.001), \mathrm{T} 90\left(27.1 \pm 4.6 \%, \mathrm{M}_{\text {diff }}=-11.2 \%, P\right.$ $<.001), \mathrm{T} 120\left(23.3 \pm 6.2 \%, \mathrm{M}_{\text {diff }}=-15.0 \%, P<.001\right)$, $\mathrm{T} 150\left(22.3 \pm 7.7 \%, \mathrm{M}_{\text {diff }}=-16.0 \%, P<.001\right)$, and $\mathrm{T} 180$ $\left(22.8 \pm 7.7 \%, \mathrm{M}_{\text {diff }}=-15.5 \%, P<.001\right)$. Monocytes significantly decreased from PRE to T90 $(9.8 \pm 1.1$ to 8.9 $\left.\pm 1.1 \mathrm{~K} / \mu \mathrm{L}, \mathrm{M}_{\text {diff }}=-0.95 \mathrm{k} / \mu \mathrm{L}, P<.001\right)$ and $\mathrm{T} 120(8.2$ $\left.\pm 1.5 \mathrm{~K} / \mu \mathrm{L}, \mathrm{M}_{\text {diff }}=-1.6 \mathrm{k} / \mu \mathrm{L}, P<.0001\right)$.

There was a significant increase in CD $34+(3.9 \pm 2.0$ to $5.3 \pm 2.8$ cells $\left.\cdot \mu \mathrm{L}^{-1}, \mathrm{M}_{\text {diff }}=1.40, P<.001\right)$ from PRE to T20 postexercise session. There were no significant differences in measured cytokine levels, i.e., IL10 , IL-6, IL-1ra, TNF- $\alpha$, or IL-2, from PRE to any time point postexercise session.

\section{Discussion}

The exercise protocol resulted in a significant elevation of WBCs with more specific analysis, suggesting an increase in peripheral HPCs, as noted in the rise of CD34+ counts and lymphocytes as noted by an automated hemocytometer. Peripheral platelet levels also immediately elevated following the exercise session. The increase in platelets we observed following the exercise session was only at time point T20 before it significantly decreased and ultimately reached near normal values 24 hours following the exercise session. This observation may illustrate a physiologic overcompensation as the individual's systemic balance affects a return to homeostasis. No discernable effect upon cytokines of interest to orthopaedics, specifically regarding inflammation and cell production, was observed.

WBC counts were examined because they are reflective of the peripheral increase of progenitor cells with stem potential. Immature cells with stem potential have one nucleus and may be read as lymphocytes or monocytes by an automated hematology analyzer. There was a significant increase in lymphocytes, and conversely, a significant decrease in average neutrophils immediately following the exercise session. The significant rise in lymphocytes and converse decrease in neutrophils may represent an increase of progenitor cells, which were registered as lymphocytes by the automated processing that was used for the $\mathrm{CBC}$ analysis; however, the peripheral changes we observed with respect to the leukocytes are also consistent with postexercise changes noted in previous studies. ${ }^{14-17}$ The converse swing we noted between the neutrophils and lymphocytes likely represents physiologic overcorrection following the exercise session as the body attempts to reachieve homeostasis, similar to the platelets.

Previous literature has demonstrated increased cytokine levels as an acute response to exercise. ${ }^{18-20}$ We chose IL-10, IL-6, GM-CSF, IL-1ra, TNF- $\alpha$, or IL-2, as the majority of these are associated with the balance of homeostasis and inflammation with joint, and GM-CSF is a potentiator of mononuclear cells. ${ }^{11,12}$ We found no significant changes in levels at any time point from PRE to 24 hours postexercise session. Ultimately, this finding may be directly attributed to the systemic stress minimizing effects of using the Vasper system during exercise. The short-time duration of exercise also could have attributed to the lack of acute cytokine level changes that were observed. Previous studies that have found significant changes in circulating cytokine levels have focused on multiple exercises, longer endurance training sessions, and were generally carried out over longer periods of time than our study. ${ }^{19-21}$ Our results, however, are consistent with previous studies that did not find a significant change in cytokine levels following exercise. ${ }^{22,23}$ Patient demographics in these studies also varied widely, however, and many times were not reflective of the participants in our study. 
Table 1. Results at Each Time Point

\begin{tabular}{|c|c|c|c|c|c|c|c|c|c|}
\hline & PRE & T20 & $\mathrm{T} 30$ & T60 & T90 & $\mathrm{T} 120$ & $\mathrm{~T} 150$ & $\mathrm{~T} 180$ & $\mathrm{~T} 24 \mathrm{H}$ \\
\hline \multicolumn{10}{|l|}{$\overline{\mathrm{WBC}}, \mathrm{K} \cdot \mu \mathrm{L}^{-1}$} \\
\hline Participant l & 6.2 & 7.3 & 5.6 & 6.1 & 3.8 & 5.8 & 6.3 & 5.8 & 5.4 \\
\hline Participant 2 & 5.2 & 6.1 & 6.1 & 4.3 & 5.6 & 5.6 & 5.2 & 5.4 & 5 \\
\hline Participant 3 & 5.5 & 5.6 & 5.6 & 4.4 & 4.4 & 4.4 & 4.4 & 4.9 & 4.8 \\
\hline Participant 4 & 4.3 & 5.1 & 4 & 3.6 & 3.7 & 4 & 3.1 & 3.9 & 4.4 \\
\hline Participant 5 & 4.7 & 8.3 & 7 & 4.2 & 3.9 & & & 4.8 & 4.4 \\
\hline Participant 6 & 6.7 & 7.3 & 5.7 & 6.3 & 6.6 & 6.6 & 6.3 & 6 & \\
\hline Participant 7 & 5 & 4.9 & 4.4 & & 4.3 & 4.5 & 6.6 & 7.1 & 4.1 \\
\hline Participant 8 & 5.6 & 8 & 5.7 & 4.6 & 2.3 & 4 & 3.8 & 5.2 & 5.8 \\
\hline Participant 9 & 5.5 & 10.6 & 10.5 & 6.2 & 5.7 & 7.4 & 9.9 & 10 & 6.3 \\
\hline Participant 10 & 8 & 10.9 & 7.6 & 5.4 & 6.1 & 8.4 & 8.6 & 8.2 & 5.9 \\
\hline Participant 11 & 6.4 & 10.2 & 6.7 & 5.7 & 5.1 & 5.3 & 6.5 & 6.3 & 6 \\
\hline Participant 12 & 5.8 & 9.3 & 8.1 & 5.7 & 6 & 6.9 & 8.9 & 8.8 & 4.8 \\
\hline Participant 13 & 7.6 & 12.1 & 9.6 & 6.6 & 6.2 & 6.5 & 7.2 & 8 & 6.3 \\
\hline Participant 14 & 6.6 & 10 & 7.7 & 6 & 7.2 & 11.5 & 11.4 & 11 & 6.8 \\
\hline Participant 15 & 8 & 13.6 & 10.6 & 7 & 6.8 & 9.1 & 9.4 & 9.2 & 6.5 \\
\hline Participant 16 & 6.4 & 12.5 & 10.8 & 5.8 & 6 & 6.4 & 7.5 & 8.2 & 6.4 \\
\hline Mean & $6.1 \pm 1.1$ & $8.8 \pm 2.7^{*}$ & $7.2 \pm 2.2^{*}$ & $5.5 \pm 1.0^{\dagger}$ & $5.3 \pm 1.3^{\dagger}$ & $6.4 \pm 2.1$ & $7.0 \pm 2.3$ & $7.1 \pm 2.1^{*}$ & $5.5 \pm 0.8^{\dagger}$ \\
\hline \multicolumn{10}{|l|}{ Platelets, $\mathrm{K} \cdot \mu \mathrm{L}^{-1}$} \\
\hline Participant 1 & 300 & 297 & 256 & 284 & 95 & 217 & 264 & 201 & 189 \\
\hline Participant 2 & 188 & 175 & 185 & 110 & 203 & 203 & 181 & 190 & 198 \\
\hline Participant 3 & 228 & 146 & 239 & 183 & 217 & 215 & 175 & 223 & 229 \\
\hline Participant 4 & 168 & 186 & 162 & 150 & 114 & 149 & 67 & 88 & 161 \\
\hline Participant 5 & 169 & 208 & 176 & 152 & 148 & & & 144 & 162 \\
\hline Participant 6 & 263 & 270 & 247 & 259 & 251 & 266 & 257 & 269 & \\
\hline Participant 7 & 206 & 213 & 192 & & 186 & 198 & 204 & 203 & 199 \\
\hline Participant 8 & 217 & 276 & 209 & 209 & & & & 152 & 217 \\
\hline Participant 9 & 231 & 299 & 281 & 252 & 250 & 238 & 239 & 209 & 241 \\
\hline Participant 10 & 192 & 222 & 184 & 167 & 164 & 174 & 173 & 165 & 173 \\
\hline Participant 11 & 176 & 218 & 179 & 176 & 151 & 174 & 171 & 167 & 186 \\
\hline Participant 12 & 265 & 296 & 287 & 258 & 234 & 252 & 271 & 257 & 87 \\
\hline Participant 13 & 261 & 349 & 305 & 249 & 237 & 255 & 239 & 256 & 262 \\
\hline Participant 14 & 233 & 267 & 234 & 218 & 208 & 210 & 202 & 203 & 200 \\
\hline Participant 15 & 272 & 312 & 279 & 259 & 236 & 237 & 170 & 238 & 256 \\
\hline Participant 16 & 393 & 503 & 484 & 364 & 387 & 371 & 381 & 364 & 367 \\
\hline Mean & $239.5 \pm 57.2$ & $268.6 \pm 86.3^{*}$ & $248.2 \pm 79.1$ & $219.3 \pm 64.9^{\dagger}$ & $206.8 \pm 72.5^{\dagger}$ & $225.6 \pm 53.7^{\dagger}$ & $213.9 \pm 71.6^{\dagger}$ & $204.0 \pm 62.9^{\dagger}$ & $208.7 \pm 61.7^{\dagger}$ \\
\hline \multicolumn{10}{|l|}{ Neutrophils, \% } \\
\hline Participant 1 & 55.4 & 58.9 & 62.1 & 69.8 & 70 & 72.1 & 70.7 & 68.7 & 55.2 \\
\hline Participant 2 & 49.4 & 41 & 47.2 & 55.9 & 60.9 & 60.9 & 64.8 & 64.9 & 53.5 \\
\hline Participant 3 & 46.6 & 41 & 45.2 & 49.5 & 54.6 & 56.4 & 56.7 & 57.6 & 52.5 \\
\hline Participant 4 & 44.9 & 48.7 & 53.5 & 55.5 & 56.1 & 54.6 & 50.4 & 50.6 & 46.5 \\
\hline Participant 5 & 43.6 & 34.4 & 36.2 & 43.1 & 48.7 & & & 47.9 & 40.8 \\
\hline Participant 6 & 46.1 & 48.9 & 52.1 & 56.2 & 57 & 57.3 & 57.8 & 54 & \\
\hline Participant 7 & 61.2 & 60.2 & 63.9 & & 66 & 68.2 & 76.9 & 79.3 & 58.5 \\
\hline Participant 8 & 48.8 & 44.5 & 47.1 & 54 & 59.3 & 69.7 & 64.4 & 67.2 & 48.3 \\
\hline Participant 9 & 49.7 & 32.9 & 35.3 & 49.2 & 65.2 & 75.6 & 77.8 & 76.1 & 45.6 \\
\hline
\end{tabular}




\begin{tabular}{|c|c|c|c|c|c|c|c|c|c|}
\hline & PRE & $\mathrm{T} 20$ & $\mathrm{~T} 30$ & $\mathrm{~T} 60$ & $\mathrm{~T} 90$ & $\mathrm{~T} 120$ & $\mathrm{~T} 150$ & $\mathrm{~T} 180$ & $\mathrm{~T} 24 \mathrm{H}$ \\
\hline Participant 10 & 43.6 & 37.5 & 42.4 & 50.9 & 64.1 & 70.2 & 68.8 & 67.7 & 41.6 \\
\hline Participant 11 & 53.8 & 47.1 & 54.6 & 58.7 & 59.7 & 61.3 & 64.2 & 64.5 & 56.3 \\
\hline Participant 12 & 46.2 & 36.9 & 41.9 & 48.5 & 55 & 65.8 & 69.5 & 70 & 48.5 \\
\hline Participant 13 & 44.3 & 38.6 & 40.9 & 50.4 & 58 & 63.2 & 63.8 & 63.4 & 49.5 \\
\hline Participant 14 & 33.3 & 28 & 32.4 & 37.7 & 68.8 & 80.5 & 81.9 & 83.1 & 43.4 \\
\hline Participant 15 & 42.1 & 31.3 & 35.8 & 54.7 & 66.5 & 71.9 & 75.8 & 77.5 & 30.4 \\
\hline Participant 16 & 56.4 & 43.4 & 48 & 62.5 & 67.8 & 71.6 & 73.7 & 75.1 & 58.5 \\
\hline Mean & $47.8 \pm 6.6$ & $42.0 \pm 9.1^{\dagger}$ & $46.2 \pm 9.3$ & $53.1 \pm 7.7^{*^{\dagger}}$ & $60.8 \pm 6.1^{*}$ & $66.6 \pm 7.5^{*}$ & $67.8 \pm 8.7^{*}$ & $68.0 \pm 9.3^{*}$ & $48.6 \pm 67.6$ \\
\hline \multicolumn{10}{|l|}{ Lymphocytes, \% } \\
\hline Participant 1 & 31.5 & 27.2 & 24 & 19.3 & 18.8 & 19.2 & 19 & 23 & 30.4 \\
\hline Participant 2 & 40.2 & 51.1 & 42.9 & 33.9 & 29.6 & 29.6 & 26.6 & 26.4 & 34.7 \\
\hline Participant 3 & 41.3 & 47.6 & 42.9 & 37.8 & 34.9 & 32.6 & 31.9 & 31.6 & 36.3 \\
\hline Participant 4 & 39.5 & 36.1 & 30.3 & 27.5 & 28.8 & 30.6 & 37.4 & 36.4 & 39.9 \\
\hline Participant 5 & 37 & 46.3 & 44.7 & 37.1 & 32.3 & & & 35.9 & 38.9 \\
\hline Participant 6 & 42 & 38.6 & 35.6 & 31.9 & 31.9 & 31.4 & 30.9 & 35.2 & \\
\hline Participant 7 & 25.3 & 27.4 & 22.8 & & 21.3 & 20.3 & 13 & 11.9 & 27.4 \\
\hline Participant 8 & 36.6 & 41.1 & 38 & 31.2 & 28.1 & 25.8 & 26.7 & 22.7 & 37.4 \\
\hline Participant 9 & 38.2 & 55.1 & 52.8 & 39.5 & 21.7 & 14.4 & 11.8 & 14.1 & 41.9 \\
\hline Participant 10 & 41.9 & 48.2 & 42.2 & 33.3 & 23.9 & 19.2 & 22 & 22.7 & 44.4 \\
\hline Participant 11 & 34.3 & 41.5 & 35.3 & 30.9 & 31 & 29 & 26.5 & 26.7 & 34.4 \\
\hline Participant 12 & 36.2 & 46.9 & 41.8 & 33.7 & 28.5 & 22.5 & 20 & 20.6 & 34.8 \\
\hline Participant 13 & 35.7 & 42.9 & 41.1 & 31.3 & 26 & 22.4 & 23.1 & 24.6 & 33.6 \\
\hline Participant 14 & 53.5 & 57.7 & 52.9 & 48.7 & 21.6 & 12.4 & 11.5 & 11.3 & 43.9 \\
\hline Participant 15 & 47.7 & 56.9 & 53.8 & 35.7 & 25.9 & 19.3 & 16.6 & 17 & 55.9 \\
\hline Participant 16 & 32.7 & 44 & 40 & 26.8 & 23.2 & 20.3 & 18 & 17.7 & 31.9 \\
\hline Mean & $38.3 \pm 6.5$ & $44.3 \pm 9.0^{*}$ & $41.2 \pm 8.2$ & $33.2 \pm 6.6^{\dagger}$ & $27.1 \pm 4.6^{\dagger}$ & $23.3 \pm 6.2^{\dagger}$ & $22.3 \pm 7.7^{\dagger}$ & $22.8 \pm 7.7^{\dagger}$ & $37.7 \pm 6.9$ \\
\hline \multicolumn{10}{|l|}{ Monocytes, $\mathrm{k} / \mu \mathrm{L}$} \\
\hline Participant 1 & 10 & 11 & 11.1 & 9.3 & 8.9 & 7.3 & 8.8 & 6.6 & 10.7 \\
\hline Participant 2 & 8.2 & 6.1 & 7.9 & 8.1 & 7.9 & 7.9 & 6.8 & 6.9 & 9.8 \\
\hline Participant 3 & 10 & 9.7 & 9.7 & 10.1 & 9 & 8.9 & 9.1 & 9.2 & 9.2 \\
\hline Participant 4 & 12.1 & 12.3 & 12.9 & 13.4 & 12.1 & 12 & 8.9 & 10.1 & 9.6 \\
\hline Participant 5 & 10.9 & 11.9 & 11.4 & 11 & 10.3 & & & 9.8 & 10.5 \\
\hline Participant 6 & 9.6 & 10.5 & 9.9 & 9.9 & 9.4 & 9.8 & 9.8 & 8.7 & \\
\hline Participant 7 & 10.5 & 9.7 & 10.4 & & 10.4 & 9.5 & 8 & 7.4 & 11 \\
\hline Participant 8 & 10.5 & 9.9 & 10.1 & 9.6 & 9.2 & 8.4 & 6.5 & 8.1 & 10.2 \\
\hline Participant 9 & 9.8 & 10.3 & 9.9 & 9.5 & 11.1 & 8.9 & 9.4 & 8.8 & 10.2 \\
\hline Participant 10 & 11.5 & 11.3 & 12.4 & 12.2 & 9.5 & 7.9 & 7.1 & 7.7 & 10 \\
\hline Participant 11 & 9.2 & 8.6 & 7.6 & 7.6 & 6.9 & 7.4 & 7.1 & 6.8 & 7 \\
\hline Participant 12 & 8.5 & 8.6 & 8.3 & 8.7 & 8.1 & 6.5 & 6.2 & 5.6 & 8.9 \\
\hline Participant 13 & 9.9 & 9.4 & 9.3 & 8.9 & 8.4 & 8.2 & 7.8 & 6.9 & 9 \\
\hline Participant 14 & 9.5 & 11.2 & 10.8 & 10.4 & 7.7 & 5.8 & 5.4 & 4.4 & 9.6 \\
\hline Participant 15 & 8.5 & 9.8 & 8.9 & 7.8 & 6.2 & 7.9 & 6.5 & 4.8 & 10.8 \\
\hline Participant 16 & 8.3 & 10.2 & 8.9 & 8.3 & 6.7 & 6.4 & 6.4 & 5.8 & 7 \\
\hline Mean & $9.8 \pm 1.1$ & $10.0 \pm 1.5$ & $10.0 \pm 1.5$ & $9.7 \pm 1.6$ & $8.9 \pm 1.6^{\dagger}$ & $8.2 \pm 1.5^{\dagger}$ & $7.6 \pm 1.3$ & $7.35 \pm 1.7$ & $9.6 \pm 1.2$ \\
\hline
\end{tabular}




\begin{tabular}{|c|c|c|c|c|c|c|c|c|c|}
\hline & PRE & $\mathrm{T} 20$ & $\mathrm{~T} 30$ & $\mathrm{~T} 60$ & T90 & $\mathrm{T} 120$ & $\mathrm{~T} 150$ & $\mathrm{~T} 180$ & $\mathrm{~T} 24 \mathrm{H}$ \\
\hline \multicolumn{10}{|l|}{ Eosinophils, \% } \\
\hline Participant 1 & 2.6 & 2.1 & 2.2 & 1.1 & 1.3 & 0.9 & 1.1 & 1.2 & 2.6 \\
\hline Participant 2 & 1 & 0.8 & 1 & 0.9 & 0.4 & 0.4 & 0.4 & 0.4 & 1 \\
\hline Participant 3 & 1.5 & 1.1 & 1.3 & 1.6 & 1.1 & 1.4 & 1.1 & 0.8 & 1 \\
\hline Participant 4 & 2.8 & 2.1 & 2.5 & 2.5 & 2.2 & 2 & 2 & 1.8 & 2.8 \\
\hline Participant 5 & 8.1 & 6.6 & 6.8 & 8.1 & 7.9 & & & 5.8 & 8.9 \\
\hline Participant 6 & 1.8 & 1.5 & 1.6 & 1.3 & 1.1 & 1.1 & 1 & 0.7 & \\
\hline Participant 7 & 2.2 & 1.9 & 2 & & 1.6 & 1.1 & 1.1 & 0.7 & 2.4 \\
\hline Participant 8 & 3.2 & 3.6 & 3.9 & 4.1 & 2.6 & 2.3 & 1.8 & 1.4 & 3.1 \\
\hline Participant 9 & 1.6 & 1.1 & 1.2 & 1.1 & 1.1 & 0.5 & 0.3 & 0.3 & 1.3 \\
\hline Participant 10 & 2.5 & 2.3 & 2.2 & 2.8 & 1.6 & 1.6 & 1.3 & 1.3 & 3.1 \\
\hline Participant 11 & 1.9 & 2 & 2.1 & 1.9 & 1.6 & 1.3 & 1.5 & 1.3 & 1.7 \\
\hline Participant 12 & 8 & 6.7 & 6.9 & 8 & 7.4 & 4.2 & 3.5 & 3.2 & 6.6 \\
\hline Participant 13 & 8.8 & 8 & 7.5 & 8.3 & 6.5 & 5.1 & 4.3 & 4.1 & 6.8 \\
\hline Participant 14 & 2.7 & 2.3 & 2.7 & 2.3 & 1 & 0.6 & 0.4 & 0.3 & 2.1 \\
\hline Participant 15 & 1 & 1 & 0.9 & 1 & 0.7 & 0.3 & 0.6 & 0.2 & 1.5 \\
\hline Participant 16 & 1.7 & 1.6 & 1.9 & 1.4 & 1.3 & 0.9 & 0.8 & 0.6 & 1.7 \\
\hline Mean & $3.2 \pm 2.6$ & $2.8 \pm 2.3^{\dagger}$ & $2.9 \pm 2.2$ & $3.1 \pm 2.7$ & $2.5 \pm 2.5$ & $1.6 \pm 1.4 \dagger$ & $1.4 \pm 1.1^{\dagger}$ & $1.5 \pm 1.6^{\dagger}$ & $3.1 \pm 2.4$ \\
\hline \multicolumn{10}{|l|}{ Basophils, \% } \\
\hline Participant 1 & 0.2 & 0.4 & 0.4 & 0.3 & 0.5 & 0.2 & 0.2 & 0.2 & 0.4 \\
\hline Participant 2 & 0.8 & 0.5 & 0.5 & 0.7 & 0.7 & 0.7 & 0.8 & 0.7 & 0.8 \\
\hline Participant 3 & 0.4 & 0.4 & 0.5 & 0.5 & 0.2 & 0.5 & 0.7 & 0.4 & 0.6 \\
\hline Participant 4 & 0.5 & 0.8 & 0.8 & 0.8 & 0.8 & 0.8 & 1 & 0.8 & 0.7 \\
\hline Participant 5 & 0.2 & 0.6 & 0.6 & 0.7 & 0.3 & & & 0.4 & 0.7 \\
\hline Participant 6 & 0.3 & 0.4 & 0.4 & 0.2 & 0.3 & 0.2 & 0.3 & 0.3 & \\
\hline Participant 7 & 0.6 & 0.6 & 0.7 & & 0.7 & 0.7 & 0.5 & 0.4 & 0.5 \\
\hline Participant 8 & 0.7 & 0.6 & 0.5 & 0.7 & 0.4 & 0.5 & 0.3 & 0.4 & 0.7 \\
\hline Participant 9 & 0.5 & 0.4 & 0.6 & 0.5 & 0.7 & 0.3 & 0.3 & 0.4 & 0.8 \\
\hline Participant 10 & 0.4 & 0.5 & 0.8 & 0.6 & 0.7 & 0.7 & 0.5 & 0.6 & 0.7 \\
\hline Participant 11 & 0.6 & 0.5 & 0.3 & 0.5 & 0.6 & 0.8 & 0.5 & 0.5 & 0.3 \\
\hline Participant 12 & 0.9 & 0.8 & 1 & 0.9 & 0.8 & 0.7 & 0.7 & 0.5 & 0.6 \\
\hline Participant 13 & 0.8 & 0.7 & 0.6 & 0.6 & 0.6 & 0.6 & 0.6 & 0.6 & 0.8 \\
\hline Participant 14 & 0.8 & 0.7 & 0.9 & 0.7 & 0.6 & 0.4 & 0.4 & 0.4 & 0.7 \\
\hline Participant 15 & 0.6 & 0.9 & 0.5 & 0.7 & 0.6 & 0.4 & 0.4 & 0.3 & 0.9 \\
\hline Participant 16 & 0.6 & 0.5 & 0.6 & 0.5 & 0.5 & 0.3 & 0.4 & 0.4 & 0.6 \\
\hline Mean & $0.56 \pm 0.22$ & $0.58 \pm 0.16$ & $0.61 \pm 0.19$ & $0.59 \pm 0.18$ & $0.56 \pm 0.18$ & $0.52 \pm 0.21$ & $0.51 \pm 0.22$ & $0.46 \pm 0.15$ & $0.65 \pm 0.16$ \\
\hline
\end{tabular}

NOTE. Mean \pm standard deviation.

WBC, white blood cells.

*Significant increase from PRE.

${ }^{\dagger}$ Significant decrease from PRE. 
Table 2. Flow Cytometry and ELISA Results at Each Time Point

\begin{tabular}{|c|c|c|c|c|c|c|c|c|c|}
\hline & PRE & $\mathrm{T} 20$ & $\mathrm{~T} 30$ & $\mathrm{~T} 60$ & $\mathrm{~T} 90$ & $\mathrm{~T} 120$ & $\mathrm{~T} 150$ & $\mathrm{~T} 180$ & $\mathrm{~T} 24 \mathrm{H}$ \\
\hline \multicolumn{10}{|l|}{$\overline{\mathrm{IL}}-1 \mathrm{RA}, \mathrm{mg} \cdot \mathrm{dL}^{-1}$} \\
\hline \multicolumn{10}{|l|}{ Participant 1} \\
\hline \multicolumn{10}{|l|}{ Participant 2} \\
\hline Participant 3 & 4284.5 & 3638.5 & 3048.4 & 3123.6 & 2760.4 & 2845.6 & 3075.0 & 2804.4 & 5401.5 \\
\hline Participant 4 & 5524.9 & 4869.8 & 5192.9 & 3517.4 & 3895.9 & 3895.9 & 4470.4 & 5202.8 & 5689.8 \\
\hline Participant 5 & 5038.9 & 6824.5 & 5857.1 & 4440.8 & 6316.6 & 6972.6 & 6861.0 & 7174.2 & 4998.0 \\
\hline Participant 6 & 7149.7 & 5699.5 & 5543.4 & 5683.9 & 6677.4 & 6455.0 & 6054.8 & 6597.8 & 4788.7 \\
\hline Participant 7 & 6831.3 & 6269.2 & 6007.6 & 5541.2 & 6431.9 & 6368.8 & 5848.4 & 4820.5 & 1889.9 \\
\hline Participant 8 & 8385.7 & $11,487.3$ & $11,596.9$ & $14,895.7$ & $13,094.9$ & $13,682.4$ & 6210.6 & $12,585.8$ & 3478.2 \\
\hline Participant 9 & 3698.3 & 5022.8 & 4037.5 & 4005.4 & 5967.1 & 4124.9 & 2055.9 & 2433.9 & 3170.1 \\
\hline Participant 10 & 3094.6 & 2981.0 & 2595.8 & 2150.5 & 2493.5 & 3502.8 & 4862.8 & 3437.6 & 2840.8 \\
\hline Participant 11 & 2671.0 & 3958.8 & 2853.4 & 2463.4 & 3064.2 & 3784.5 & 3781.3 & 3388.4 & 3136.7 \\
\hline Participant 12 & 5167.8 & 3785.3 & 3605.5 & 3377.2 & 3376.3 & 3502.8 & 4178.3 & 4594.2 & \\
\hline Participant 13 & 2458.1 & 2530.8 & 2584.7 & 2348.2 & 3035.2 & 3189.7 & 2112.2 & 2152.6 & 1899.6 \\
\hline Participant 14 & 7777.6 & 8076.2 & 7448.8 & 7494.6 & 8923.4 & 9074.0 & 8559.8 & 6091.6 & 6018.5 \\
\hline Participant 15 & 2068.3 & 2672.9 & 2474.3 & 2230.2 & 2112.2 & 2271.5 & 1931.0 & 1669.1 & 1830.4 \\
\hline Participant 16 & 2659.9 & 2902.6 & 2556.7 & 2523.4 & 2772.9 & 1986.5 & 2150.4 & 2400.3 & 2270.5 \\
\hline Mean & $4772.2 \pm 2121.8$ & $5051.4 \pm 2501.7$ & $4671.6 \pm 2562.1$ & $\begin{array}{r}4556.8 \pm \\
3365.0\end{array}$ & $\begin{array}{r}5065.8 \pm \\
3094.1\end{array}$ & $\begin{array}{r}5118.3 \pm \\
3187.2\end{array}$ & $4439.4 \pm 2070.8$ & $4668.1 \pm 2870.5$ & $3647.1 \pm 1541.3$ \\
\hline \multicolumn{10}{|l|}{$\mathrm{IL}-10, \mathrm{mg} \cdot \mathrm{dL}^{-1}$} \\
\hline \multicolumn{10}{|l|}{ Participant 1} \\
\hline \multicolumn{10}{|l|}{ Participant 2} \\
\hline Participant 3 & 296.3 & 311.1 & 333.5 & 364.6 & 332.3 & 329.9 & 259.3 & 287.3 & 437.3 \\
\hline Participant 4 & 417.1 & 406.2 & 425.0 & 412.3 & 435.4 & 435.4 & 406.5 & 425.4 & 453.0 \\
\hline Participant 5 & 375.2 & 568.8 & 513.4 & 502.7 & 535.7 & 507.7 & 560.6 & 586.2 & 532.5 \\
\hline Participant 6 & 628.1 & 552.8 & 533.1 & 519.2 & 634.5 & 596.0 & 542.8 & 552.7 & 339.0 \\
\hline Participant 7 & 388.2 & 388.2 & 396.5 & 346.3 & 379.7 & 412.1 & 396.9 & 434.6 & 415.5 \\
\hline Participant 8 & 375.2 & 388.2 & 419.4 & 489.0 & 578.4 & 463.2 & 470.7 & 549.5 & 485.6 \\
\hline Participant 9 & 512.9 & 542.8 & 666.1 & 565.5 & 756.2 & 546.0 & 292.4 & 406.4 & 628.3 \\
\hline Participant 10 & 338.9 & 294.8 & 213.4 & 216.9 & 310.7 & 342.7 & 189.9 & 281.3 & 437.4 \\
\hline Participant 11 & 386.8 & 344.0 & 320.1 & 338.9 & 310.7 & 445.9 & 456.5 & 518.7 & 425.7 \\
\hline Participant 12 & 438.6 & 473.9 & 472.5 & 438.6 & 462.6 & 438.6 & 557.7 & 559.9 & \\
\hline Participant 13 & 214.2 & 189.9 & 225.0 & 214.2 & 225.0 & 189.9 & 177.2 & 202.6 & 214.2 \\
\hline Participant 14 & 277.2 & 236.6 & 212.3 & 214.2 & 257.5 & 267.1 & 277.2 & 236.6 & 225.0 \\
\hline Participant 15 & 175.5 & 225.8 & 246.7 & 214.2 & 272.2 & 277.2 & 212.3 & 189.9 & 189.9 \\
\hline Participant 16 & 295.0 & 303.5 & 303.5 & 265.4 & 355.3 & 295.9 & 295.0 & 267.6 & 276.2 \\
\hline Mean & $365.7 \pm 116.7$ & $373.3 \pm 124.2$ & $377.2 \pm 137.2$ & $364.4 \pm 157.7$ & $417.6 \pm 3094.1$ & $396.2 \pm 116.4$ & $363.9 \pm 138.0$ & $392.8 \pm 145.5$ & $389.2 \pm 132.4$ \\
\hline \multicolumn{10}{|l|}{$\mathrm{IL}-6, \mathrm{mg} \cdot \mathrm{dL}^{-1}$} \\
\hline \multicolumn{10}{|l|}{ Participant 1} \\
\hline \multicolumn{10}{|l|}{ Participant 2} \\
\hline Participant 3 & 213.8 & 222.7 & 202.9 & 203.6 & 178.3 & 163.9 & 226.8 & 173.8 & 311.1 \\
\hline Participant 4 & 266.5 & 282.5 & 269.3 & 211.3 & 273.2 & 273.2 & 281.8 & 288.9 & 303.5 \\
\hline Participant 5 & 235.6 & 357.3 & 303.4 & 266.3 & 370.2 & 371.0 & 380.3 & 383.3 & 292.8 \\
\hline Participant 6 & 390.3 & 339.2 & 326.4 & 365.6 & 345.0 & 391.1 & 361.0 & 367.2 & 293.5 \\
\hline Participant 7 & 458.5 & 359.2 & 388.9 & 391.3 & 457.1 & 403.7 & 435.9 & 292.2 & 152.0 \\
\hline Participant 8 & 979.9 & 783.2 & 1090.7 & 1038.7 & 1149.4 & 995.5 & 514.5 & 1199.1 & 232.5 \\
\hline
\end{tabular}


Table 2. Continued

\begin{tabular}{|c|c|c|c|c|c|c|c|c|c|}
\hline & PRE & $\mathrm{T} 20$ & $\mathrm{~T} 30$ & T60 & T90 & $\mathrm{T} 120$ & $\mathrm{~T} 150$ & $\mathrm{~T} 180$ & $\mathrm{~T} 24 \mathrm{H}$ \\
\hline Participant 9 & 283.6 & 273.1 & 298.3 & 219.5 & 214.5 & 253.9 & 92.5 & 191.2 & 288.2 \\
\hline Participant 10 & 235.0 & 230.0 & 184.1 & 133.1 & 197.8 & 255.6 & 102.5 & 171.7 & 252.5 \\
\hline Participant 11 & 224.0 & 217.1 & 175.6 & 176.5 & 294.8 & 340.5 & 310.9 & 325.0 & 270.5 \\
\hline Participant 12 & 224.0 & 211.5 & 262.7 & 236.0 & 235.7 & 272.9 & 313.5 & 347.2 & \\
\hline Participant 13 & 134.9 & 120.9 & 156.9 & 181.4 & 177.6 & 148.7 & 130.4 & 140.0 & 130.4 \\
\hline Participant 14 & 157.5 & 165.6 & 213.5 & 279.1 & 261.3 & 279.1 & 353.3 & 301.5 & 110.1 \\
\hline Participant 15 & 130.4 & 207.0 & 181.4 & 258.7 & 180.5 & 181.4 & 208.6 & 119.7 & 86.7 \\
\hline Participant 16 & 195.5 & 206.1 & 189.0 & 185.3 & 236.5 & 140.0 & 188.6 & 187.4 & 119.7 \\
\hline Mean & $295.0 \pm 216.8$ & $283.9 \pm 159.9$ & $303.1 \pm 236.7$ & $296.2 \pm 225.2$ & $326.6 \pm 250.5$ & $319.3 \pm 213.3$ & $278.6 \pm 126.7$ & $320.6 \pm 267.7$ & $218.7 \pm 85.1$ \\
\hline $\begin{array}{l}\mathrm{IL}-2, \mathrm{mg} \cdot \mathrm{dL}^{-1} \\
\text { Participant } 1 \\
\text { Participant } 2\end{array}$ & & & & & & & & & \\
\hline Participant 3 & 357.7 & 415.8 & 404.0 & 427.9 & 391.4 & 372.8 & 383.6 & 346.9 & 496.5 \\
\hline Participant 4 & 516.6 & 520.9 & 520.9 & 458.0 & 512.6 & 512.6 & 508.7 & 509.8 & 540.0 \\
\hline Participant 5 & 469.6 & 622.8 & 579.4 & 538.8 & 614.5 & 540.9 & 638.4 & 642.9 & 566.8 \\
\hline Participant 6 & 593.4 & 570.9 & 473.1 & 558.7 & 599.2 & 546.0 & 532.2 & 570.5 & 388.6 \\
\hline Participant 7 & 578.5 & 553.5 & 540.0 & 441.5 & 581.1 & 599.6 & 578.5 & 528.6 & 425.1 \\
\hline Participant 8 & 765.0 & 740.8 & 856.8 & 1055.4 & 940.7 & 939.4 & 616.2 & 1047.8 & 541.1 \\
\hline Participant 9 & 650.6 & 671.3 & 670.4 & 651.6 & 674.4 & 739.6 & 300.4 & 494.2 & 589.7 \\
\hline Participant 10 & 280.3 & 290.8 & 224.2 & & 251.6 & 298.1 & & 224.2 & 398.8 \\
\hline Participant 11 & 375.1 & 268.2 & 260.9 & 188.9 & 268.2 & 412.4 & 375.7 & 431.6 & 451.8 \\
\hline Participant 12 & 398.8 & 398.3 & 417.0 & 375.1 & 407.1 & 441.8 & 519.6 & 591.0 & \\
\hline Participant 13 & 250.5 & 220.6 & 278.4 & 259.8 & 240.8 & 240.8 & 220.0 & 250.5 & 230.7 \\
\hline Participant 14 & 322.3 & 313.7 & 287.7 & 292.2 & 296.3 & 312.9 & 296.3 & 278.4 & 287.7 \\
\hline Participant 15 & 240.4 & 260.2 & 287.7 & 278.4 & 287.1 & 296.6 & 275.7 & 220.0 & 208.7 \\
\hline Participant 16 & 333.2 & 330.7 & 313.7 & 287.1 & 354.7 & 320.9 & 278.7 & 322.3 & 285.8 \\
\hline Mean & $438.0 \pm 161.4$ & $441.3 \pm 170.2$ & $436.7 \pm 182.4$ & $447.2 \pm 227.0$ & $458.5 \pm 204.1$ & $469.6 \pm 195.1$ & $424.9 \pm 145.8$ & $461.3 \pm 221.7$ & $416.2 \pm 130.2$ \\
\hline \multicolumn{10}{|l|}{$\mathrm{TNF}-\alpha, \mathrm{mg} \cdot \mathrm{dL}^{-1}$} \\
\hline \multicolumn{10}{|l|}{ Participant 1} \\
\hline \multicolumn{10}{|l|}{ Participant 2} \\
\hline Participant 3 & 136.7 & 167.9 & 146.0 & 168.6 & 142.8 & 139.5 & 107.6 & 126.8 & 180.6 \\
\hline Participant 4 & 208.2 & 209.4 & 226.8 & 198.1 & 214.4 & 214.4 & 188.7 & 182.8 & 215.9 \\
\hline Participant 5 & 175.9 & 263.6 & 238.8 & 258.4 & 264.3 & 241.2 & 271.7 & 246.6 & 245.3 \\
\hline Participant 6 & 230.3 & 215.5 & 197.9 & 208.0 & 257.7 & 223.9 & 209.7 & 231.9 & 136.5 \\
\hline Participant 7 & 194.3 & 168.4 & 162.6 & 145.7 & 169.2 & 168.9 & 191.2 & 150.0 & 136.5 \\
\hline Participant 8 & 163.7 & 193.5 & 231.7 & 196.7 & 228.2 & 240.7 & 192.0 & 320.0 & 252.3 \\
\hline Participant 9 & 260.8 & 269.4 & 291.6 & 235.4 & 286.3 & 291.0 & 121.3 & 197.8 & 275.6 \\
\hline Participant 10 & 100.1 & 109.8 & 84.5 & 82.2 & 117.3 & 124.3 & 68.5 & 92.7 & 138.8 \\
\hline Participant 11 & 152.0 & 124.3 & 83.2 & & 117.3 & 190.2 & 158.4 & 194.0 & 158.4 \\
\hline Participant 12 & 170.7 & 176.5 & 164.4 & 176.7 & 179.4 & 190.7 & 230.3 & 215.0 & \\
\hline Participant 13 & 73.7 & 54.8 & 78.9 & 83.0 & 60.2 & 59.7 & 48.5 & 60.2 & 48.5 \\
\hline Participant 14 & 91.1 & 78.9 & 70.0 & 70.0 & 82.5 & 87.0 & 87.2 & 65.1 & 74.4 \\
\hline Participant 15 & 36.7 & 54.8 & 54.8 & 49.4 & 65.1 & 70.0 & 75.7 & 54.8 & 49.4 \\
\hline Participant 16 & 101.8 & 101.8 & 90.7 & 78.6 & 109.6 & 90.7 & 77.6 & 74.4 & 74.4 \\
\hline Mean & $149.7 \pm 63.5$ & $156.3 \pm 70.9$ & $151.6 \pm 76.4$ & $150.1 \pm 69.9$ & $163.9 \pm 72.3$ & $166.6 \pm 72.7$ & $144.9 \pm 70.0$ & $158.0 \pm 82.0$ & $152.8 \pm 77.9$ \\
\hline CD $34+$ cells $\cdot \mu \mathrm{L}^{-1}$ & & & & & & & & & \\
\hline
\end{tabular}




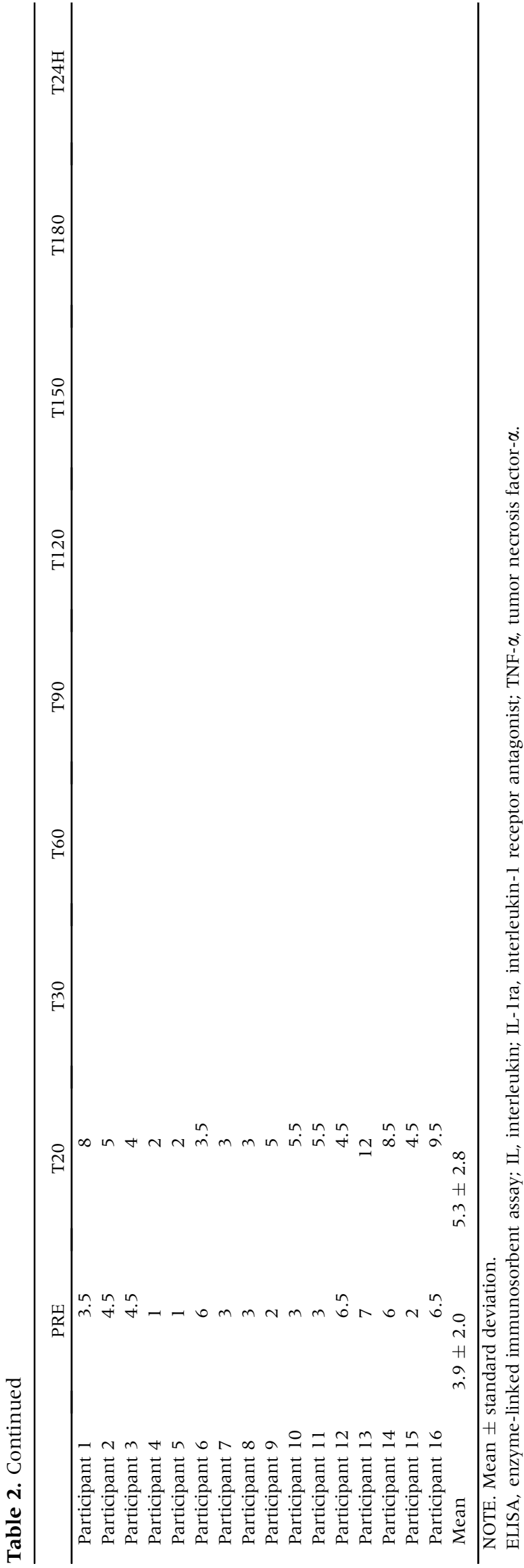

Ultimately expanding the cytokine and growth factor analysis to include IL-3, monocyte chemoattractant protein-1, insulin-like growth factor-1, insulin-like growth factor-2, growth hormone, sIL-1R, and soluble tumor necrosis factor receptors may provide further data of the Vasper system on both pro- and antiinflammatory cytokines and growth factors. In addition, it is important to consider the individual variability in blood levels as well as the variability in blood levels at different time points in the same individual.

The Vasper system was designed to produce highintensity anaerobic exercise with a low impact on joints. The NuStep is frequently used in rehabilitation because its low-impact nature of exercise, which patients with lower-extremity orthopaedic impairments can tolerate. The blood flow restriction and cooling properties of the Vasper system provide additional modalities to increase the intensity of exercise. A cellular response of increased peripheral CD34+ and platelets was observed following exercise with the Vasper system. If available, it may be used as a way to manipulate point-of-care blood products, especially the yield of platelet-rich plasma immediately following exercise. Anz et al. ${ }^{8}$ reported that vigorous exercise for 20 minutes increased the concentration of platelets in whole blood by more than $20 \%$ and in buffy coat-based platelet-rich plasma and autologous condition plasma point-of-care products. Buffy coat-based platelet-rich plasma also had significantly increased HPCs from $1.7 / \mathrm{mL}$ to $2.7 / \mathrm{mL}$ following exercise. Bone marrow aspiration and peripheral bloodstream harvest after pharmaceutical mobilization represent 2 of the current methods for collecting HPCs. ${ }^{3,24}$ Pharmaceutical mobilization followed by apheresis can produce large volumes of peripheral blood-derived stem cells with 600 thousand HPCs per milliliter and 2.32 million perivascular stem cells per milliliter of tissue collected. ${ }^{25}$ Although HPCs hold promise for a variety of orthopaedic applications, it has yet to be determined what is the optimal collection technique. ${ }^{7,26}$ Both bone marrow aspiration and pharmaceutical mobilization have limitations in their application to widespread clinical use.

Exercise could be a potentially less-invasive method to mobilize stem cells to optimize the physiology of recovering orthopaedic patients as well as to manipulate point-of-care blood and bone marrow products in orthopaedics. Previous literature has also demonstrated variability in platelet product yield among commercially available platelet rich plasma kits. ${ }^{27}$ The Vasper system may be potentially leveraged as a way to increase peripheral platelet release prior to blood draw to improve the platelet-rich plasma yield that would be administered, as exercise in itself has already been found as a method to manipulate these products. ${ }^{8}$ 


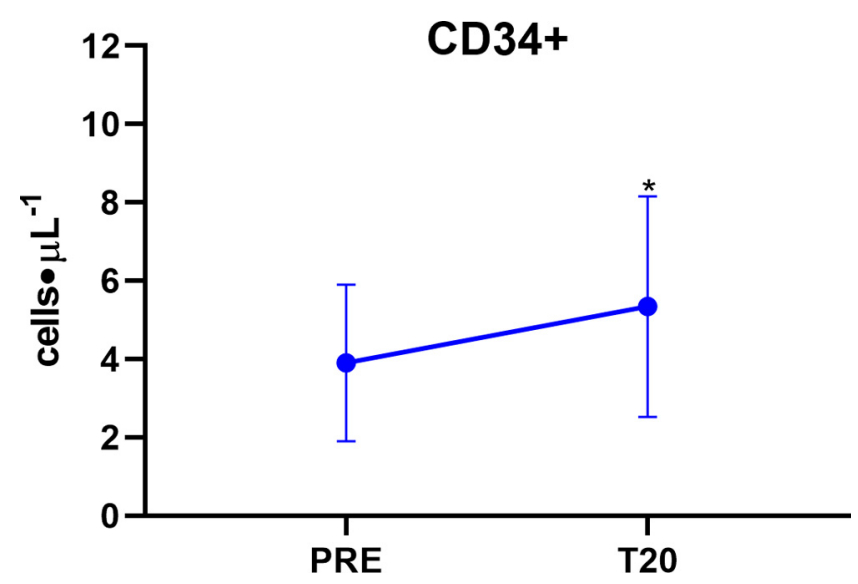

Fig 3. The graphed trends of the mean and standard deviation of CD34+ pre- and postexercise session.

\section{Limitations}

Flow cytometry was used to investigate CD34+ levels only at the time point T20 due to the cost and time associated with using flow cytometry for every time point. We hypothesized that measuring levels immediately postexercise would give the best single point of data on whether peripheral levels of CD34+ increased following the Vasper exercise session. This limits knowledge regarding whether CD34+ levels remained elevated, decreased, or returned to baseline in the immediate postexercise window and up to 24 hours later. Only healthy, male participants were included in this study; thus, the results may not be generalizable to other individuals. Another limitation of this study includes the lack of a control group to identify whether the postexercise systemic cellular changes noted were the result of the exercise stimulus alone or if the exercise stimulus with the Vasper system would produce different results. It is possible that similar cellular changes would have occurred with the same exercise protocol that did not include compression and cooling. This study was self-funded, and the costs associated with performing flow cytometry and ELISA testing limited our ability to include a control session to the protocol. Another limitation is that although the amount of pressure applied by the cuffs on the arms and legs was the same for each participant, varying levels of occlusion may have occurred between participants based on their blood pressure and limb girth.

\section{Conclusions}

A significant elevation of peripheral blood CD34+ and platelet levels immediately following the exercise session was observed; however, there was no effect upon peripheral circulation of IL-10, IL-6, GM-CSF, ILlra, TNF- $\alpha$, or IL-2.

\section{Acknowledgments}

The Vasper System was donated to the researchers by Vasper.

\section{References}

1. Meheux CJ, McCulloch PC, Lintner DM, Varner KE, Harris JD. Efficacy of intra-articular platelet-rich plasma injections in knee osteoarthritis: A systematic review. Arthroscopy 2016;32:495-505.

2. Holig K, Kramer M, Kroschinsky F, et al. Safety and efficacy of hematopoietic stem cell collection from mobilized peripheral blood in unrelated volunteers: 12 years of single-center experience in 3928 donors. Blood 2009;1 14:3757-3763.

3. Smith JN, Calvi LM. Concise review: Current concepts in bone marrow microenvironmental regulation of hematopoietic stem and progenitor cells. Stem Cells 2013;31: 1044-1050.

4. Anz AW, Branch EA, Rodriguez J, et al. Viable stem cells are in the injury effusion fluid and arthroscopic byproducts from knee cruciate ligament surgery: An in vivo analysis. Arthroscopy 2017;33:790-797.

5. Morikawa D, Johnson JD, Kia C, et al. Examining the potency of subacromial bursal cells as a potential augmentation for rotator cuff healing: An in vitro study. Arthroscopy 2019;35:2978-2988.

6. Callanan MC, Plummer HA, Chapman GL, et al. Blood flow restriction training using the Delfi System is associated with a cellular systemic response. Arthrosc Sports Med Rehabil 2021;3:e189-e198.

7. Rochefort GY, Delorme B, Lopez A, et al. Multipotential mesenchymal stem cells are mobilized into peripheral blood by hypoxia. Stem Cells 2006;24:2202-2208.

8. Anz AW, Parsa RS, Romero-Creel MF, et al. Exercisemobilized platelet-rich plasma: Short-term exercise increases stem cell and platelet concentrations in plateletrich plasma. Arthroscopy 2019;35:192-200.

9. Emmons R, Niemiro GM, Owolabi O, De Lisio M. Acute exercise mobilizes hematopoietic stem and progenitor cells and alters the mesenchymal stromal cell secretome. J Appl Physiol (1985) 2016;120:624-632.

10. Marycz K, Mierzejewska K, Smieszek A, et al. Endurance exercise mobilizes developmentally early stem cells into peripheral blood and increases their number in bone marrow: Implications for tissue regeneration. Stem Cells Int 2016;2016:5756901.

11. Shephard RJ, Rhind S, Shek PN. Exercise and training: Influences on cytotoxicity, interleukin-1, interleukin-2 and receptor structures. Int $J$ Sports Med 1994;15: S154-S166 (suppl 3).

12. Shephard RJ, Rhind S, Shek PN. The impact of exercise on the immune system: NK cells, interleukins 1 and 2, and related responses. Exerc Sport Sci Rev 1995;23:215-241.

13. Keser I, Suyani E, Yosmaoglu HB, Aki SZ, Turkoz Sucak AG. Acute physiological responses to physiotherapy applications pre and post autologous stem cell transplantation: An experimental study. Hematology 2014;19:136-140.

14. Kim K, Suzuki K, Peake J, et al. Physiological and leukocyte subset responses to exercise and cold 
exposure in cold-acclimatized skaters. Biol Sport 2014;31:39-48.

15. Sand KL, Flatebo T, Andersen MB, Maghazachi AA. Effects of exercise on leukocytosis and blood hemostasis in 800 healthy young females and males. World J Exp Med 2013;3:11-20.

16. Peake JM, Neubauer O, Walsh NP, Simpson RJ. Recovery of the immune system after exercise. J Appl Physiol (1985) 2017;122:1077-1087.

17. Rooney BV, Bigley AB, LaVoy EC, Laughlin M, Pedlar C, Simpson RJ. Lymphocytes and monocytes egress peripheral blood within minutes after cessation of steady state exercise: A detailed temporal analysis of leukocyte extravasation. Physiol Behav 2018;194:260-267.

18. Moldoveanu AI, Shephard RJ, Shek PN. The cytokine response to physical activity and training. Sports Med 2001;31:115-144.

19. Kimura H, Suzui M, Nagao F, Matsumoto K. Highly sensitive determination of plasma cytokines by time-resolved fluoroimmunoassay; Effect of bicycle exercise on plasma level of interleukin-1 alpha (IL-1 alpha), tumor necrosis factor alpha (TNF alpha), and interferon gamma (IFN gamma). Anal Sci 2001;17:593-597.

20. Suzuki K, Nakaji S, Yamada M, Totsuka M, Sato K, Sugawara K. Systemic inflammatory response to exhaustive exercise. Cytokine kinetics. Exerc Immunol Rev 2002;8:6-48.

21. Ostrowski K, Schjerling P, Pedersen BK. Physical activity and plasma interleukin-6 in humans-effect of intensity of exercise. Eur J Appl Physiol 2000;83:512-515.
22. Ferreira FC, de Medeiros AI, Nicioli C, et al. Circuit resistance training in sedentary women: Body composition and serum cytokine levels. Appl Physiol Nutr Metab 2010;35:163-171.

23. Libardi CA, De Souza GV, Cavaglieri CR, Madruga VA, Chacon-Mikahil MP. Effect of resistance, endurance, and concurrent training on TNF-alpha, IL-6, and CRP. Med Sci Sports Exerc 2012;44:50-56.

24. Vangsness CT Jr, Sternberg H, Harris L. Umbilical cord tissue offers the greatest number of harvestable mesenchymal stem cells for research and clinical application: A literature review of different harvest sites. Arthroscopy 2015;31:1836-1843.

25. Schmitz N, Bacigalupo A, Hasenclever D, et al. Allogeneic bone marrow transplantation vs filgrastim-mobilised peripheral blood progenitor cell transplantation in patients with early leukaemia: First results of a randomised multicentre trial of the European Group for Blood and Marrow Transplantation. Bone Marrow Transplant 1998;21: 995-1003.

26. Saw KY, Anz A, Siew-Yoke Jee C, et al. Articular cartilage regeneration with autologous peripheral blood stem cells versus hyaluronic acid: A randomized controlled trial. Arthroscopy 2013;29:684-694.

27. Fitzpatrick J, Bulsara MK, McCrory PR, Richardson MD, Zheng MH. Analysis of platelet-rich plasma extraction: Variations in platelet and blood components between 4 common commercial kits. Orthop J Sports Med 2017;5: 2325967116675272 . 